\title{
Holography: origin, basic principle and applications of a revolutionary communication method in art and science
}

\author{
Giorgio Matteucci \\ Department of Physics and Astronomy - University of Bologna, V/le B. Pichat, Bologna, Italy
}

\begin{abstract}
History of human kind shows that man has developed various communication methods such as language, art, writing, photography, etc. The most revolutionary of these methods is holography, a completely new way to look at the world around us. Here we describe how art, writing and holography, that at a first hasty analysis might be considered different unrelated disciplines, have actually an interesting common background. This include the basic men's need to transfer information as well as the contribution of a few important actors who developed the information transfer process. A short account is set of the origin and the working principle of holography against this multi-disciplinary framework. Although a large number of applications of holography has been devised, here only one example is reported to demonstrate the potential of electron holography to display pictorial maps of magnetic lines of force in matter.
\end{abstract}

Key words: Optical holography, electron holography, magnetic domains, magnetic flux lines.

Holografía: origen, principio básico y aplicaciones de un método de comunicación revolucionario en arte y ciencia

Resumen

La historia de la humanidad muestra que el hombre ha desarrollado varios métodos de comunicación como el lenguaje, el arte, la escritura, la fotografía, etc. El más revolucionario de estos métodos es la holografía, una nueva manera de mirar el mundo que nos rodea. En este artículo se describe cómo el arte, la escritura y la holografía, que en un primer análisis apresurado podrían considerase como disciplinas diferentes y no relacionadas entre sí, tienen en realidad un interesante fondo común. Esto incluye la necesidad básica del hombre de transferir información, como también la contribución de algunos actores importantes que desarrollaron el proceso de transferencia de información. Brevemente se establecen el origen y principio de funcionamiento de la holografía frente a este marco multidisciplinar. Aunque ha sido inventado un gran numero de aplicaciones de la holografía, solo reportaremos aquí un ejemplo quedemuestra el potencial de la holografía de electrones para desplegar mapas pictoricos de líneas de fuerza magnetica en la materia.

Palabras clave: holografía óptica, holografía de electrones, dominios magnéticos, líneas de flujo magnético

\section{Introduction}

Imagine you are the first person entering the Altamira caves discovered in Spain or the Lascaux ones in France. Prehistoric graffiti representing an animal will unexpectedly appear in front of you. It was to favour his hunting that primitive man made the first graffiti and afterwards the first paintings inside the caverns where he lived. Very suggestive evidence was also found in central and south America as well as in the Fumane cave in Italy, almost certainly the world's oldest ones.

In 1799 close to the ruin of Fort Rashid near the River Nile a grey granite stone, later world wide known as the Stele of Rosetta, was recovered and is now at the British Museum. Due to damage caused by the elements, the central engravings are partly unreadable. Further down, 54 lines in Greek and Greek symbols are engraved. In the upper part, 14 lines in antique Egyptian hieroglyphics, figures of human beings, birds, etc. are reproduced. It turned out that an interpretation of the message through the symbols only allowed a limited comprehension level to be reached. A developed language must be able to report suitably about facts belonging to every day life, to give a full account of logical connections and describe satisfactory abstract concepts. Both in the case of prehistoric graffiti and Egyptian hieroglyphics we are faced with messages to decipher whose correct interpretation

\footnotetext{
Corresponding author:

Giorgio Matteucci, giorgio.matteucci@unibo.it

Recibido: 30 de junio de 2015

Aceptado: 9 de septiembre de 2015
} 
open the way towards a deeper understanding of the spirit which animated our forefathers or, even more simply, a clear understanding of the techniques used to transmit information. While in the case of the depicting of animals the message can have a fairly rapid explanation, it was far more difficult conceptually and time consuming to decode the hieroglyphics of the Stele of Rosetta.

The reader can rightly wonder what all this has to do with holography and its possibility to disclose the peculiarities of nature. It is interesting to note that two of the really brilliant minds in the last two hundred years gave a more or less direct contribution to the decoding of the information to be found on the Stele of Rosetta. One of these men was Thomas Young, born in Milverton, U.K. in 1773, six years before the finding of the Stele. A man of far-reaching cultural ideas, he was interested in mathematics and physics and graduated in medicine at Gottingen in 1795. In his lectures read before the Royal Society he supported the wave theory of light and implemented it with a new basic concept, the principle of interference. In 1814 it seems that some friends reminded Young of the problems connected with the Stele of Rosetta. Up to 1819 Young made good progress in the understanding of that document called triglot (there were three texts) but a number of reasons seem to have prevented him from doing much more to complete the undertaking. The person who contributed to solving the problem even though not directly, was the famous French mathematician, Jean Baptiste Joseph, Baron de Fourier, who was born in 1768 and had taken part in the Napoleonic expedition to Egypt as scientific adviser. Like Young, he too had a 'wave vision of nature', regarding the universe as a great organ which oscillated in a complicated way. The understanding of the single phenomena of the physical world could then be reached by trying to reduce this particular gigantic oscillation into thousands of elementary harmonic oscillations that existed in clear determined ratios. In 1801 Fourier presented the 'mystery of the Stele' to a very bright precocious fifteen year old, Francois Champollion. With incredible persistence, difficult to find in someone so young, in 1821 Champollion was able to decipher the famous document.

We have seen how two great men were able to contribute in some way to decoding the information obtained from incisions, that is, from elements which are part of reality and can be perceived directly with our main sensorial organ, the eye. In the following we will describe how, differently from the eye, holography is able to display a three-dimensional image of an object or, alternatively, maps of magnetic fields in matter. The holographic method is based fundamentally on two distinct stages (Gabor, 1948; Hariharan, 2002; Johnston, 2006). In the first, the information regarding the size to be examined is 'frozen' in an interference pattern of which Young could be considered the discoverer. As we shall see in the second stage, the information is decoded and processed utilizing the known properties of the Fourier transform. After the sharpened stone chisel used to engrave the walls of the caves, the use of more sophisticated technological methods, like hammer and iron chisel inaugurated the Hellenistic period first and then the Renaissance, during which works of extraordinary worldwide acknowledged beauty were passed down in history. In the great surprising changes in the history of art which took place in Greece around the 6th century BC, the Greek artists, differently from the Egyptians, tried to improve the scenes bearing in mind how these were intended to communicate more incisively, the three-dimensional perspective both in painting and in sculpture. At the beginning of the 15th century a scientific approach to the art world was opened by Filippo Brunelleschi who discovered the geometric rules of perspective representation (Prager, et al., 1970). Based on mathematical laws, Brunelleschi's method allowed artists to realize two-dimensional pictures which were suggested as the most faithful imitation possible of nature and of the work accomplished by man. A completely new way to look at nature came into being. Young and Fourier, with their cultural interests and their careful precise analysis of physical phenomena had started to pave the way towards the discovery of holography. Since the Hellenistic age 2500 years passed before finally the most revolutionary method of visual communication was conceived. This was holography able to bring to our eyes 3-D images identical to those we perceive in the world we live in (Johnston, 2006). The extraordinary capacity holograms possess to get us to see the third dimension of a scene justifies and substantiates their utility in its most spectacular use, art. It is less known that the motivations which led Dennis Gabor in 1948 to develop the theory of holography have their basis in the deep interest he had for electron microscopy (Gabor, 1948). He knew that the fundamental resolution limit of a transmission electron microscope was due to spherical aberration which, since the theoretical work of Scherzer, was unavoidable with electron lenses (Scherzer, 1936). Gabor had probably experienced a check-mate situation, as regards the improvement of an electron microscope performance, as perhaps seen by Abbe for the light microscope. In Gabor's own words: “The fundamental development of the subject has now reached a stage beyond which progress is likely to be slow and difficult. At this point, it seems appropriate to look into the future and try to explore in imagination the avenues of further development. As this is a proverbially risky undertaking, the author is quite prepared to join the ranks of other, more illustrious prophets who have failed.” (Mulvey, 1995). Gabor exploited his "immense cerebral informationgathering and processing ability” (Mulvey, 1995) to find a novel way to overcome the practical resolution limit of the electron microscope, which at that time was about $1.2 \mathrm{~nm}$, with the correction of lens aberrations and, in particular, of the spherical one. A success along this line would have led to an improvement of the microscope's performance and the possibility to obtain a resolution down to atomic level. The 
interesting story of how Gabor dealt with this problem is described by T. Mulvey who collaborated with Gabor and was the first together with M.E. Haine to record an electron hologram. For this reason we would like to report Mulvey's own words: "For many years Gabor struggled unsuccessfully with this problem, (that of spherical aberration, author's note), but could not get it out of his mind" (Mulvey, 1995). In the Easter holidays of April 1947 Gabor reported to have had, quite unexpectedly, a 'vision' in which “... instantaneously and without effort on my part, the complete solution of the aberration correction problem was presented to me" (Mulvey, 1995). According to Gabor the vision ran as follows: “...Take an electron image that contains the whole information (i.e., amplitude and phase) and then correct it by optical means. To record amplitude and phase, a coherent background must be supplied by the electron source. The result will be an interferogram. Photograph this and then illuminate the interferogram with coherent optical light and record the reconstructed image on a photographic plate." (Mulvey, 1995). This new imaging method was named by Gabor 'holography' from the Greek o $\lambda$ os (olos) meaning 'whole' and rpapsw (grafein) meaning 'to write'. “A hologram (the interferogram) would therefore record, on a single medium such as a photographic plate, the complete electron wave in amplitude and phase. No one had ever even dreamed of this before, and the term became his exclusive trademark" (Mulvey, 1995). At that time the coherence of electron sources was too poor to record reliable holograms so that electron holography entered a stage of hibernation. The invention of the laser contributed to optical reconstruction of electron holograms and mainly determined the wide spreading of optical holography delighting neophytes with three-dimensional visions of reconstructed images. A Fresnel biprism was used to separate the wave which travelled through the object from the one passing outside it (Leith \& Upatnieks, 1962). This was called reference wave. Electron holography stood aloof for about 30 years until reliable coherent field emission sources became commercially available. A comprehensive survey of these events which led to the achievement of atomic resolution as envisaged by Gabor is reported in (Lichte, 1991). Here, I recall the basic principle of holography avoiding a mathematical treatment. Optical holography is now well established as a display medium and it is used in medicine, engineering, architecture, retailing, art etc. In particular, a large number of applications has been possible thanks to the development of holographic interferometry that enables the static and dynamic displacements of an optical rough surface to be measured (for a detailed treatment of the principles, techniques and application of optical holography see, (Hariharan, 2002; Jones \& Wykes, 1989; Vikram, 1982; Johnston, 2006; Johnston, 2009) and references therein. It is also worth mentioning the interesting results regarding digital in-line holographic light microscopy developed to observe, with wavelength resolution, 3-D representations of the paths followed by micro objects such as suspensions of microspheres and biological samples (cells, bacteria, etc.) and microchannel flow, motion of bubbles in microfluidic applications (Restrepo \& García-Sucerquia, 2012).

In view of the multidisciplinary aspect of this article and by considering the peculiar mysterious wave behaviour of material particles, attention is given to outline the working principle of electron holography. As it is well known, Michel Faraday, in order to convey to the mind a useful pictorial idea of the magnetic flux, had devised the magnetic lines of force. Here, I will report how electron holography allows to display the existence of these lines. A new method is thus available for the investigation of magnetic field configurations in matter. In particular, the observation of the magnetization distribution, in a thin nickel film, as a function of temperature is reported. Impressive images show the decreasing of the spontaneous magnetization when the temperature approaches Curie point (Matteucci, 2006).

\section{Materials and methods}

In this chapter, the working principle of light optical holography is schematically recalled (Hariharan, 2002; Johnston, 2006) and then extended to the case of electrons. The transfer of information with the holographic method takes place with a three step process, (i) an interference pattern, named hologram; is recorded on a photographic plate; (ii) the photographic process of the hologram is carried out and finally, (iii) synthesis or descramble of information is performed. Figure 1a shows schematically the well known Young arrangement to produce an interference pattern. $\mathrm{S}$ is a coherent source of light, 1 and 2 are two circular apertures on an otherwise absorbing screen. In the plane P interference fringes are obtained whose visibility is related to the coherence of the source. Let us introduce now, along the path of one of the two waves of figure $1 \mathrm{a}$, an object $\mathrm{O}$. The wave scattered from the sample, called object wave, will undergo a phase shift with respect to the wave coming from hole 1 . This last wave is named reference wave. The interference patterns recorded with this method are off-axis holograms because the object and the reference wave do not travel along the same direction as originally proposed by Gabor, but are tilted at a slight angle. While in a standard imagerecording process (i.e. photography) the phase information is lost, through a 'Young type experiment' both amplitude and phase of the object are 'frozen' in the interference fringes. Synthesis or descramble of information is performed by illuminating the developed plate DP containing the hologram with a reconstruction wave RW having the same inclination as the reference beam during the hologram recording, figure 1b. The intensity distribution of the interference pattern acts as a diffraction grating for the incoming light. Behind the plate the illuminating wave emerges and, as a consequence, also the object wave spreads out of the hologram and is seen by the eye $\mathrm{E}$ as a 3-D reconstructed image RI, identical to the 3-D object. A hologram behaves like a window 

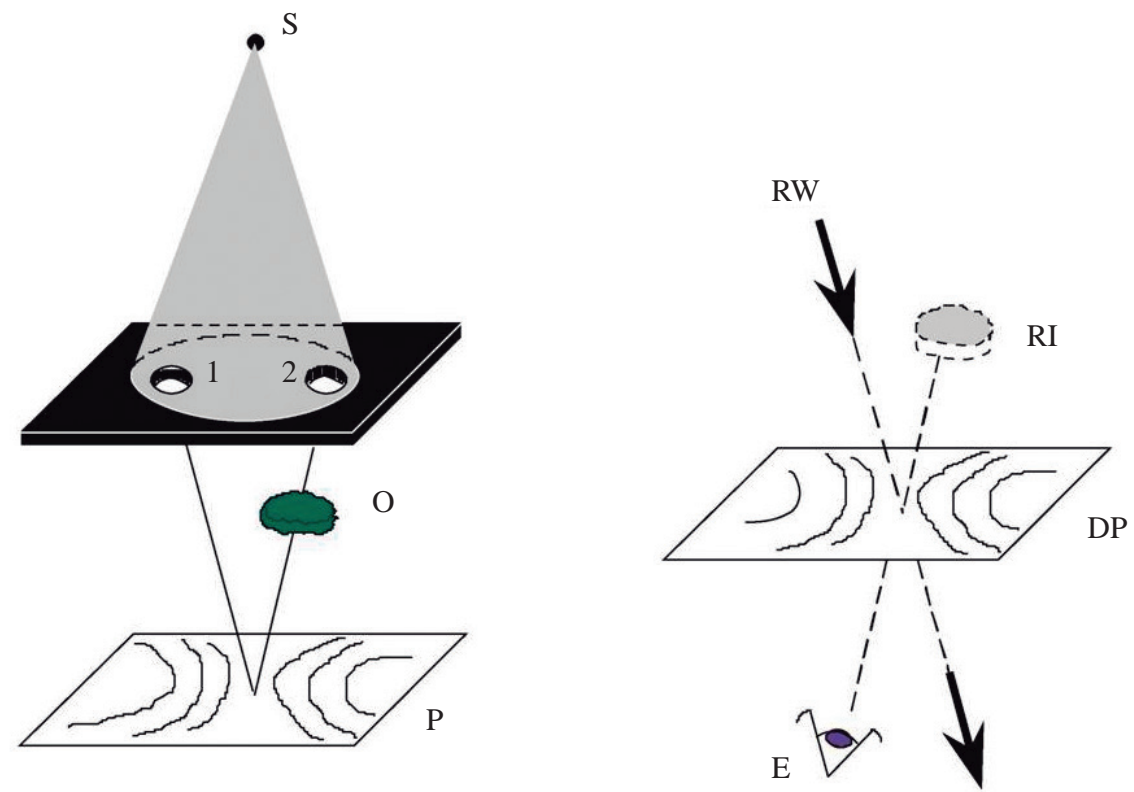

Figure 1. Outlines of the Young interferometer. (a) S, coherent light source; 1 and 2, apertures in a metal screen; O, object; P, observation plane. (b) schematic reconstruction of the object wavefront. RW, reconstruction wave; DP, developed plate; E, observer's eye; RI, reconstructed image of the object of (a).

through which the object can be observed. These general considerations, although very schematic, are still valid for radiations of any wavelength and also most important for matter wave. Moreover, the wavelength of the radiation or of particles used for holographic recording can be many orders of magnitude different from that employed for image reconstruction.

Now the recording and processing of electron holograms are briefly reviewed. These holograms are recorded mainly by means of an electron microscope equipped with an electrostatic biprism (Moellenstedt \& Dueker, 1956) The biprism is realized by placing a thin charged wire $\mathrm{W}$ between two grounded plates, figure 2 , and is located below the back focal plane of the objective lens (not drawn for simplicity). The wire splits the wave-front of the incoming coherent electron beam EB and its electrostatic field produces a deflection and a subsequent overlapping, in the observation plane OP, of that part of the wave which has travelled through the object (object wave Ow) and the other part of the wave which has passed outside the sample (reference wave Rw). Because the width of the overlapping region and the interference fringe spacing can be controlled by varying the charge on the wire, with an external voltage supply, it is possible to observe holograms on OP and to record them on a photographic plate PP or with a CCD camera. If the interference fringes are superimposed on an in-focus image of the specimen, then this interferogram is named offaxis image electron hologram. As shown in figure 2, the standard arrangement uses only one half of the object plane for the specimen $\mathrm{S}$, illuminated by $\mathrm{EB}$, while the reference

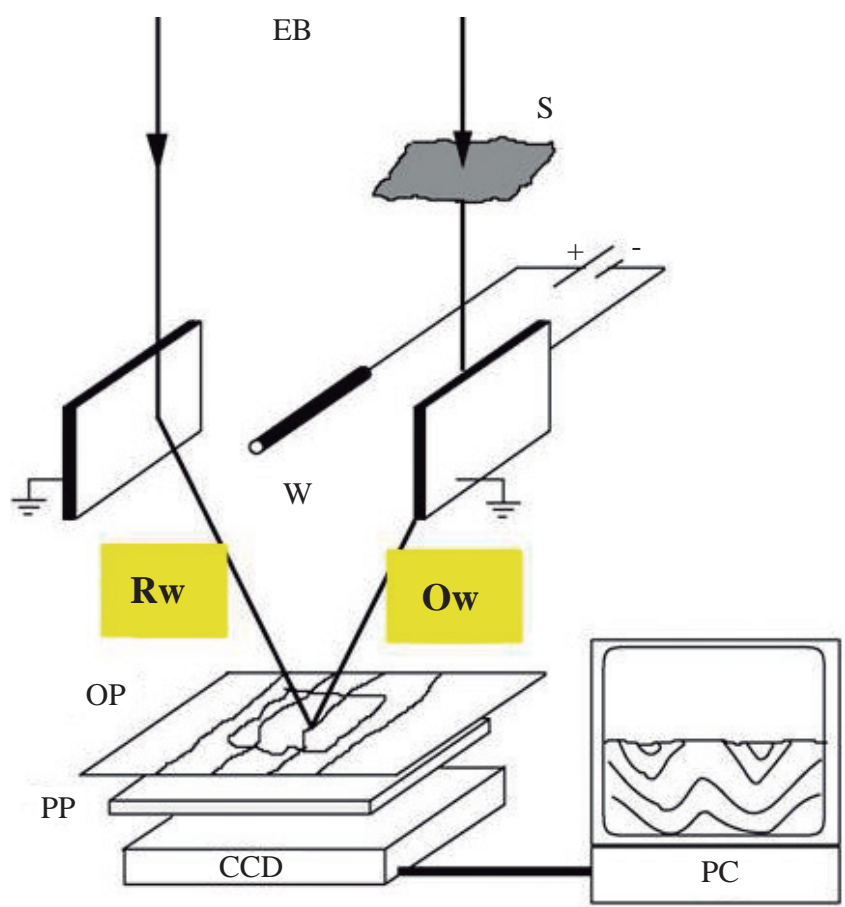

Figure 2. Realization of off-axis electron holography. EB, electron beam; S, specimen; W, biprism central wire; Rw, reference wave; Ow, object wave; OP, observation plane; PP, photographic plate; CCD, CCD camera connected to a computer for hologram processing.

beam goes through the other half plane. The recovering of information contained in the hologram can be realized by optical or digital means. These latter methods are generally 
used because of the great advantages they offer with respect to optical techniques (for a detailed description of electron holography and its applications see, Tonomura, 1999; Tonomura, et al., 1995; Voelkl, et al., 1999; Matteucci, et al., 2002; Lichte \& Lehmann, 2008). However, we will briefly describe the optical method for historical reasons, since it was used in the pioneer work on electron holography, but mainly for the instructional purposes of this article. The plate on which an electron hologram is recorded is first developed and then inserted in an optical bench and illuminated by a coherent laser wave, figure 3 . In the hologram $\mathrm{H}$ an in-focus image of the object (the image of a frog is used only for pictorial purpose) is superimposed to an interference fringe system. This is basically a co-sinusoidal grating which causes the diffraction of the laser beam LB incident on it. Generally speaking, from the plane of the hologram the wave propagates and at each subsequent point in space it can be determined by means of the Kirchhoff integral. The size of the optical system is reduced when a lens $\mathrm{L}$ (reconstruction lens) is located behind the hologram $\mathrm{H}$ as reported in figure 3 . In the back focal plane of $\mathrm{L}$ three beams are focused which are the Fourier transform of the intensity distribution of the hologram $\mathrm{H}$. In addition to the straight through beam $<0>$, two sidebands are displayed which represent the Fourier spectrum of the image wave i.e. $<1>$ and its conjugate $<-1>$. The hologram fringe spacing must be small enough to obtain the separation of the three beams in the focal plane of $\mathrm{L}$ where the $<1>$ sideband is isolated by means of a spatial filter F. Allowing a free propagation of the wave from the focal plane onwards, the inverse Fourier transform is performed on a viewing screen $\mathrm{V}$ where an exact optical replica of the object wave function is obtained which can be subsequently processed by well established optical techniques. This is the ingenuity and originality of Gabor's idea. As suggested by Gabor, for a reconstruction of a high resolution image wave, the compensation of the aberrations could be performed using a suitable phase plate inserted in the back focal plane of L. However, this is a very challenging task because of the inherent difficulty in preparing a suitable correction plate matching precisely all the parameters related to the wave aberrations (Tonomura, 1999; Lichte \& Lehmann, 2008). Today, however, image reconstruction by numerical methods presents considerable advantages with respect to the light optical technique and the effects of aberration correction at high resolution has been demonstrated by numerical image processing of digitized holograms (Lichte \& Lehmann, 2008). In this case the Fourier transform of the hologram is taken and a sideband which corresponds to the object wave is chosen. From this wave function the lens aberration term can be eliminated in principle by multiplication with the inverse of the aberration phase factor. The amplitude and phase of the true object wave can be finally obtained by a subsequent Fourier transform (Lichte, et al., 2009). In this way, Gabor's original idea to overcome the resolution limit of an electron microscope has been realized. Using off-axis holography in an aberration-corrected transmission electron microscope a resolution of a few picometers has been demonstrated (Harada, et al., 2004; Akashi, et al., 2015). However, for our purposes, only low resolution holograms are used so that the whole processing of holograms is greatly simplified.

To convey a general idea of the potential of electron holography for the investigation of electromagnetic field in matter, the process of image formation in an electron microscope is briefly reviewed together with the method of holographic interferometry (Hariharan, 2002) used to extract the information content of the electron wave which has gone through the sample under investigation. For a detailed treatment of the material reviewed in this section see references (Tonomura, 1999; Tonomura, et al., 1995; Voelkl, et al., 1999; Matteucci, et al., 2002; Lichte \& Lehmann, 2008). The process of image formation in an electron microscope is schematically described by assuming that, (i) electrons propagate as a plane wave from the source to the specimen, (ii) electrons go through the specimen, propagate through the microscope lenses and are detected by a photographic plate or an in-line electronic image read-out device. By considering only

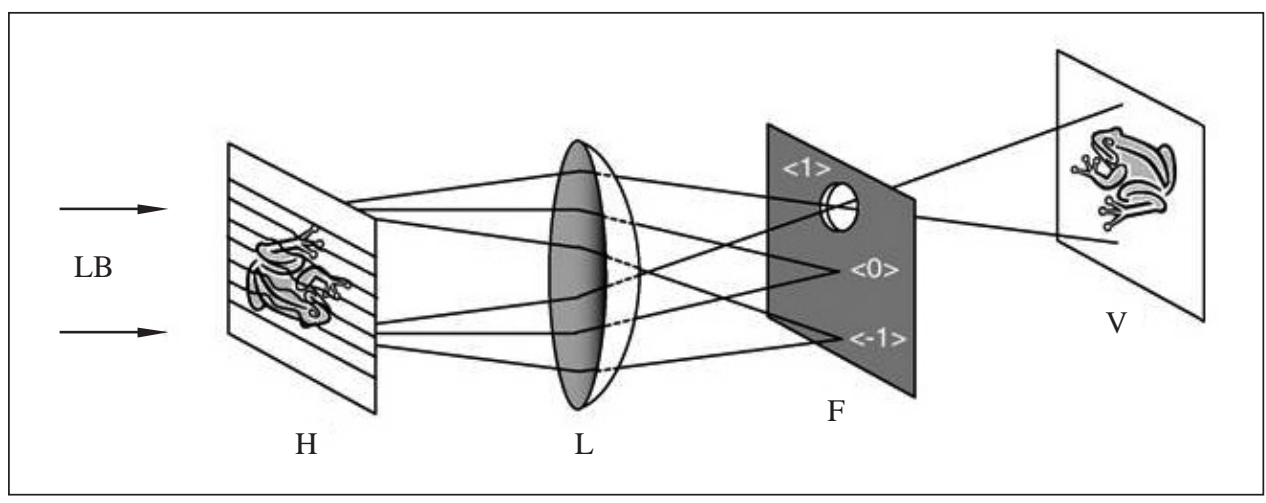

Figure 3. Diagram for hologram reconstruction. H, electron hologram illuminated by a parallel laser beam, LB; L, reconstruction lens; F, filtering aperture; $\mathrm{V}$, viewing screen. 
elastic scattering events, the interaction of the electron beam with the specimen can be described through a complex transmission function $\mathrm{O}(\mathrm{r})$. In the standard phase object approximation, $\mathrm{O}(\mathrm{r})=\exp [\mathrm{i} \phi(\mathrm{r})]$, where $\mathrm{r}=(\mathrm{x}, \mathrm{y})$ is a 2-dimensional vector perpendicular to the optic axis $\mathrm{z}$ which is parallel and in the same direction as the electron beam. The phase term $\phi(r)=\pi / \lambda E \int V(r, z) d z-2 \pi \mathrm{e} / \mathrm{h} \int \mathrm{A}_{\mathrm{z}}(\mathrm{r}, \mathrm{z})$ $\mathrm{dz}$. The integrals are taken along trajectories parallel to the optical axis $\mathrm{z}$ inside and outside the specimen to include stray fields. $\mathrm{V}(\mathrm{x}, \mathrm{y}, \mathrm{z})$ and $\mathrm{A}_{\mathrm{z}}(\mathrm{x}, \mathrm{y}, \mathrm{z})$ are, respectively, the electrostatic potential and the $\mathrm{z}$ component of the vector potential. $\lambda, \mathrm{h}$, e and $\mathrm{E}$ are, respectively, the electron wavelength, the Planck constant, the absolute value of the electron charge and the accelerating voltage in the non-relativistic approximation. Since the information is mainly encoded in the phase, it is important to recall that, contrary to the optical case where 3-dimensional effects are strikingly impressive, only essential 2-dimensional information is available in transmission electron microscopy. In fact in the electric case, $\phi$ is proportional to the potential averaged along the electron path whereas, in the magnetic case, the maximum information encoded in the beam corresponds to the magnetic flux enclosed between two electron trajectories. The most widespread use of electron holography is in the study of electric and magnetic fields down to a nanoscale (Tonomura, et al., 1995; Tonomura, 1999; Voelkl, et al., 1999; Matteucci, et al., 2002; Lichte, \& Lehmann, 2008; Dunin-Borkowski, et al., 2004; Park, et al., 2014). This possibility was not even envisaged by Gabor. Although the reconstruction of a wave front can be completely achieved by holographic technique the eye or a detector, placed in the plane $\mathrm{V}$ of figure 3, records only the intensity of the object wave function while the phase is once again lost. To recover the phase information content, holographic interferometry technique is used. It is based on the interferometric comparison of two or more waves of which, at least one, is holographically reconstructed. As schematically shown in figure $4 \mathrm{a}$ the reconstructed object wave front ROW is made to interfere with a plane wave PW while both waves propagate in the same direction. The new interference pattern obtained under this condition is named contour map, figure $4 \mathrm{~b}$. Its basic feature is that the contour lines, overlapping to the reconstructed image of the object (not shown), connect points in the sample which have suffered the same phase shift. In optics, the contour lines are representative of the changes of the physical quantities of the object under investigation such as rotation, vibration, temperature and position variations, etc. In electron holography the contour maps are interpreted in terms of electric or magnetic field distributions accordingly to the kind of sample under investigation. For example, when the sample is chemically homogeneous, diamagnetic and of varying thickness, the contour fringes are lines of constant thickness.

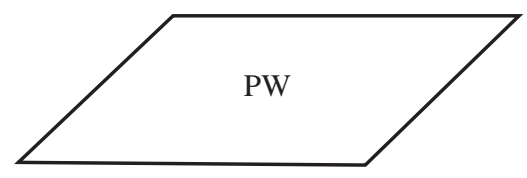

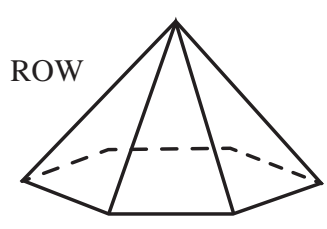

a

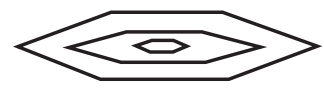

b
Figure 4. Illustration of the working principle of holographic interferometry. (a) PW, plane wave; ROW, reconstructed object wavefront;. (b) contour map resulting from the interference between ROW and PW.

\section{Results}

Although electron holography has been applied to obtain maps of electric and magnetic fields in thin films (Matteucci, et al., 2002; Harada, et al., 2004; Lichte, et al., 2007; Lichte, \& Lehmann, 2008; Lichte, et al., 2009; Park, et al., 2014); here we report only an example regarding the study of the change of the magnetization versus temperature in a thin film (Matteucci, 2006). Let us consider a magnetic sample of constant thickness and transparent to electrons. An electron wave which interacts with a this specimen suffers local phase shifts $\phi=2 \pi \mathrm{eF} / \mathrm{h}$ which depend on the magnetic flux F enclosed between interfering electron trajectories. Those electrons which have crossed the specimen at different points and have suffered the same phase shift with respect to the reference wave embrace a constant magnetic flux. The loci of points which have undergone the same phase shift are displayed as lines in the contour map, as schematically shown in figure $4 \mathrm{~b}$. A phase difference $\Delta \phi=2 \pi$ between two adjacent contour lines is produced by an enclosed magnetic flux, $\mathrm{h} / \mathrm{e}=4.1 \times 10^{-15} \mathrm{~Wb}$. Therefore, the contour fringes are directly interpreted as magnetic flux lines. A qualitative and quantitative interpretation of the contour maps of magnetic specimens is thus obtained. For a suitable interpretation of the experimental images it is worthwhile recalling that, for a first approximation, the magnetic properties of a sample can be schematically described by assuming the existence of small regions, called domains, each spontaneously magnetized. As shown in figure 5, a sample may consist of domains $\mathrm{D}$, with different magnetization directions indicated by the arrows, separated by narrow regions (thick black lines) named domain walls, DW. Across the walls the magnetization changes its direction. Figure 6 reports the contour images of the same area of a thin nickel film at various temperatures. The rim of the specimen runs almost horizontally and divides the image into two parts. The investigated area of the sample is confined in the half 


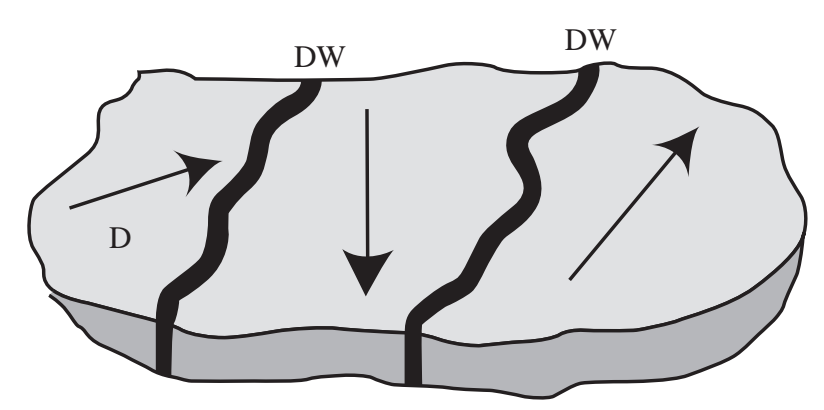

Figure 5. Schematic magnetization arrangement in a thin film. D, magnetic domains; DW, domain walls. The arrows in the domains show the magnetization directions.
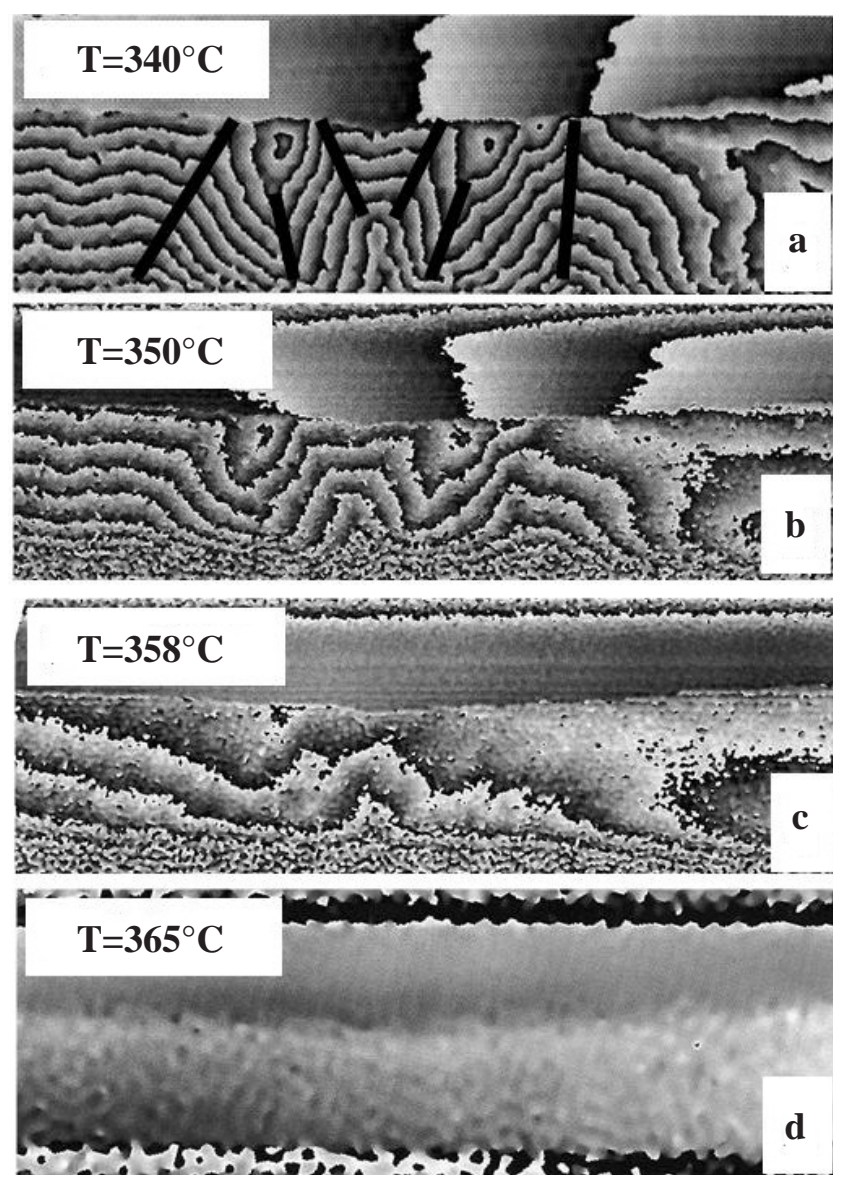

Figure 6. Contour maps of the magnetic flux lines in a thin nickel film at various temperature, (a) $340^{\circ} \mathrm{C}$; (b) $350^{\circ} \mathrm{C}$; (c) $358^{\circ} \mathrm{C}$; (d) $365^{\circ} \mathrm{C}$. All images are shown in the same scale: $7 \mathrm{~mm}=1 \mu \mathrm{m}$.

lower part where a number of bright and dark bending lines are visible. Six black, thick straight lines have been drawn to indicate the position and trend of six magnetic walls. Above the specimen rim there is a vacuum region, through which the reference wave propagates. The white wavy lines or alternatively the black ones, can be interpreted as magnetic flux lines through the specimen or, instead, as leakage field lines outside it (only two of them are visible above the rim of the sample). Between two adjacent lines a flux $\mathrm{h} / \mathrm{e}$ is enclosed. Therefore, by counting the number of lines in a given region of the sample the total magnetic flux through that area can be evaluated. The location and shape of the magnetic domain walls of the sample is revealed where the direction of the magnetization lines changes abruptly. As is well-known, the magnetization of a sample, produced without the action of an external magnetic field, is called spontaneous magnetization. As the temperature increases, the arrangement of the spins is affected by thermal movement which results in a decrease of the spontaneous magnetization in accordance with the Weiss-Curie law. At a certain temperature, named Curie point, the arrangement of the spins becomes completely random and the spontaneous magnetization vanishes. Figure $6 \mathrm{a}$, shows the trend of the magnetization at $340^{\circ} \mathrm{C}$, (the precision of the heating stage is $1^{\circ} \mathrm{C}$ ). At this temperature, the magnetic flux through the sample is slightly decreased with respect to that of room temperature. Figure $6 \mathrm{~b}$ shows that a strong decrease of the magnetization of about $50 \%$ (the number of the magnetic flux lines through the same area halves) takes place when the sample temperature reaches $350^{\circ} \mathrm{C}$. A further reduction of the magnetization occurs at $358^{\circ} \mathrm{C}$, figure $6 \mathrm{c}$, close to the Curie transition temperature (for a bulk sample is about $358^{\circ} \mathrm{C}$ ). At this temperature only a couple of magnetic lines are still visible. Finally, figure $6 \mathrm{~d}$ shows clearly that no magnetic field line is displayed when the sample temperature is $365^{\circ} \mathrm{C}$, i.e. a few degrees above the Curie point. The transition of the sample from the ferromagnetic to the paramagnetic state has taken place. It is worthwhile mentioning that also with standard Lorentz electron microscopy techniques it is possible to observe the washing out of the domain walls. However the advantage of the holographic method allows a direct visualization of the magnetization direction through the domains and a measurement of the remaining flux around the Curie point. As an example, on the left part of figure $6 \mathrm{c}$, three white lines or alternatively three dark ones are revealed. Since between two successive lines a flux $\mathrm{h} / \mathrm{e}$ is enclosed, the remanent flux through that area of the sample is $\mathrm{F}=3 \mathrm{~h} / \mathrm{e}$, i.e. about $12 \times 10^{-15} \mathrm{~Wb}$. From the knowledge of the magnetic flux F, the thickness $t$ of the sample (about 50nm), the width $\mathrm{w}$ of the sample area (about $2 \mu \mathrm{m}$ ), the average value of the magnetic field is estimated, $B=F / w t \approx 0.1 T$.

\section{Conclusions}

In the celebration of the International Year of Light 2015 an approach to electron holography has been presented keeping an eye open to possible interconnections between two, so it would seem, uncorrelated fields as those of the fine arts and science where the Young and Fourier contributions play important roles. In the same way as painters use appropriate brush strokes to represent the particulars of the scenes they want to record, holography makes use of the 'Young lines', that is lines of different intensity 
and curvature where the whole information of the wave coming from the object is stored. Fourier mathematical methods are subsequently applied to extract the whole information content of the object wave. An unusually wideranging subject, holography has attracted competing interpretations of intellectual novelty and technological application. A collaborations between artists and scientists has stimulated the use of this technique artistically as witnessed by the museum collections and exhibits from 1964 to the end of the twentieth century (Johnston, 2006; Johnston, 2009). As a case study, we have presented an application of electron holography not even envisaged by Gabor. It regards the possibility to display magnetic flux lines in materials in analogy with the magnetic lines of force devised by Faraday to convey a full and pictorial idea of the magnetic field to the mind. The study of, (i) the magnetic field around micro tips used in magnetic force microscopy (Matteucci, et al., 1994; Matteucci, et al., 2004), (ii) the magnetic field in magnetostactic bacteria (Dunin-Borkoski, et al., 1998), (iii) two-dimensional arrays of naturally occurring magnetite crystals in minerals etc., (Dunin-Borkowski, et al., 2004) are a few further examples showing how electron holography may contribute to study magnetic field configurations down to nanoscale.

\section{Conflict of interests}

The author declares no conflicts of interests.

\section{References}

Akashi, T., Takahashi, Y., Tanigaki, T., Shimakura, T., Kawasaki, T., Furutsu, T., Shinada, H., Muller, H., Haider, M., Osakabe, N., and Tonomura, A. 2015. Aberration corrected 1.2-MV cold field-emission transmission electron microscope with a sub-50-pm resolution. Appl. Phys. Lett. 106: 074101.

Dunin-Borkowski, R.E., Kasama, T., Wei, A., Tripp, S.L., Hÿtch, M.J., Snoeck, E., Harrison, R.J., Putnis, A. 2004. Off-axis electron holography of magnetic nanowires and chains, rings, and planar arrays of magnetic nanoparticles. Microscopy research and technique. Wiley Subscription Services, Inc., A Wiley Company. 64: 390-402.

Dunin-Borkoski, R.E., McCartney, M.R., Frankel, R.B., Bazylinski, D,A., Posfai, M., Buseck, P.R. 1998. Magnetic microstructure of magnetotactic bacteria by electron holography. Science. 282: 1868-1870.

Gabor, D. 1948. A new microscopic principle. Nature. 161: 181183.

Harada, K., Tonomura, A., Matsuda, T., Akashi, T., Togawa, Y. 2004. High-resolution observation by double biprism holography. J.Appl. Phys. 96: 6097-6102.

Hariharan, P. 2002. Basic of holography. Cambridge University Press.

Johnston, S. F. 2006. Holographic Visions: A History of New Science. Oxford University Press.
Johnston, S. F. 2009. Representing Holography in Museum Collections. Illuminating Instruments. Smithsonian University Press, Washington DC. Ch 6: 97-116.

Jones R. \& Wykes, C. 1989. Holographic and Speckle Interferometry. Cambridge University Press.

Leith, E.N. \& Upatnieks, Y. 1962. Reconstructed wavefronts and communication theory. J. Opt. Soc. Am. 52: 1123-1130.

Lichte, H. 1991. Image plane off-axis electron holography of atomic structures. Advances in optical and electron microscopy. Academic, London. 12: 25-91.

Lichte, H., Geiger D., Linck, M. 2009. Off-axis electron holography in an aberration-corrected transmission electron microscope. Phil. Trans. R. Soc. A 367: 3773-3793.

Lichte, H., Formanek, P., Lenk, A., Linck, M., Matzeck, C., Lehmann, M., Simon, P. 2007. Electron Holography: Applications to Materials Questions. Annu. Rev. Mater. Res. 37: 539-588.

Lichte, H. \& Lehmann, M. 2008. Electron holography-basics and applications. Reports on Progress in Physics. 71: 016102.

Matteucci, G. 2006. Direct observation of the spontaneous magnitazation near the Curie point of a thin nickel film by electron holography. Phil. Mag. Lett. 86: 81-87.

Matteucci, G., Frost, B.G., Medina, F. 2004. Study of the field around magnetic force microscopy probes using electron holography. Ultramicroscopy. 99: 95-102.

Matteucci, G., Missiroli G.F., Pozzi, G. 2002. Electron holography of long-range electrostatic fields. Advances in Imaging and Electron Physics. Elsevier Science, USA. 122: 173-249.

Matteucci, G., Muccini, M., Hartmann, U. 1994. Flux measurements on ferromagnetic microprobes by electron holography. Physical Review B: 50: 6823-6828.

Moellensted, G., Dueker, H. 1956. Beobachtungen und messungen an biprisma-interferenzen mit electronenwellen. Z. Physik. 145: 377-397.

Mulvey, T. 1995. Gabor pessimistic 1942 view of electron microscopy and how he stumbled on the Nobel Prize. Advances in Imaging and Electron Physics (Academic Press). 91: 259-283.

Park, H. S., Yu, X., Aizawa, S., Tanigaki, T., Akashi, T., Takahashi, T., Matsuda, T., Kanazawa, N., Onose, Y., Shindo, D., Tonomura, A., and Tokura, Y. 2014. Observation of the magnetic flux and three-dimensional structure of skyrmion lattices by electron holography. Nature Nanotech. 9: 337-342.

Prager, F.D., Scaglia G. 1970. Brunelleschi: Studies of His Technology and inventions. Cambridge, Mass.: M.I.T. Press.

Restrepo, J.F., García-Sucerquia, J. 2012. Automatic threedimensional tracking of particles with high numerical aperture digital lensless holographic microscopy. Optics Letters. 37 (4): 752-754.

Scherzer, O. 1936. Ueber einige Fehler von Electronenlinsen. Z. Physik. 101: 593-598. 
Tonomura, A. 1999. Electron Holography. 2nd Ed. SpringerVerlag, Berlin Heidelberg.

Tonomura, A., Allard, L.F., Pozzi, G., Joy, D.C., Ono, Y.A. 1995. Electron Holography. Elsevier, Amsterdam. (The authors are editors of this book which collects articles of different authors).
Vikram, C.S. 1982. Particle Field Holography. Cambridge University Press.

Voelkl, E., Allard, L.F., \& Joy, D.C. 1999. Introduction to Electron Holography. Kluwer Academic/Plenum Publishers, NY. (The authors are editors of this book which collects articles of different authors).

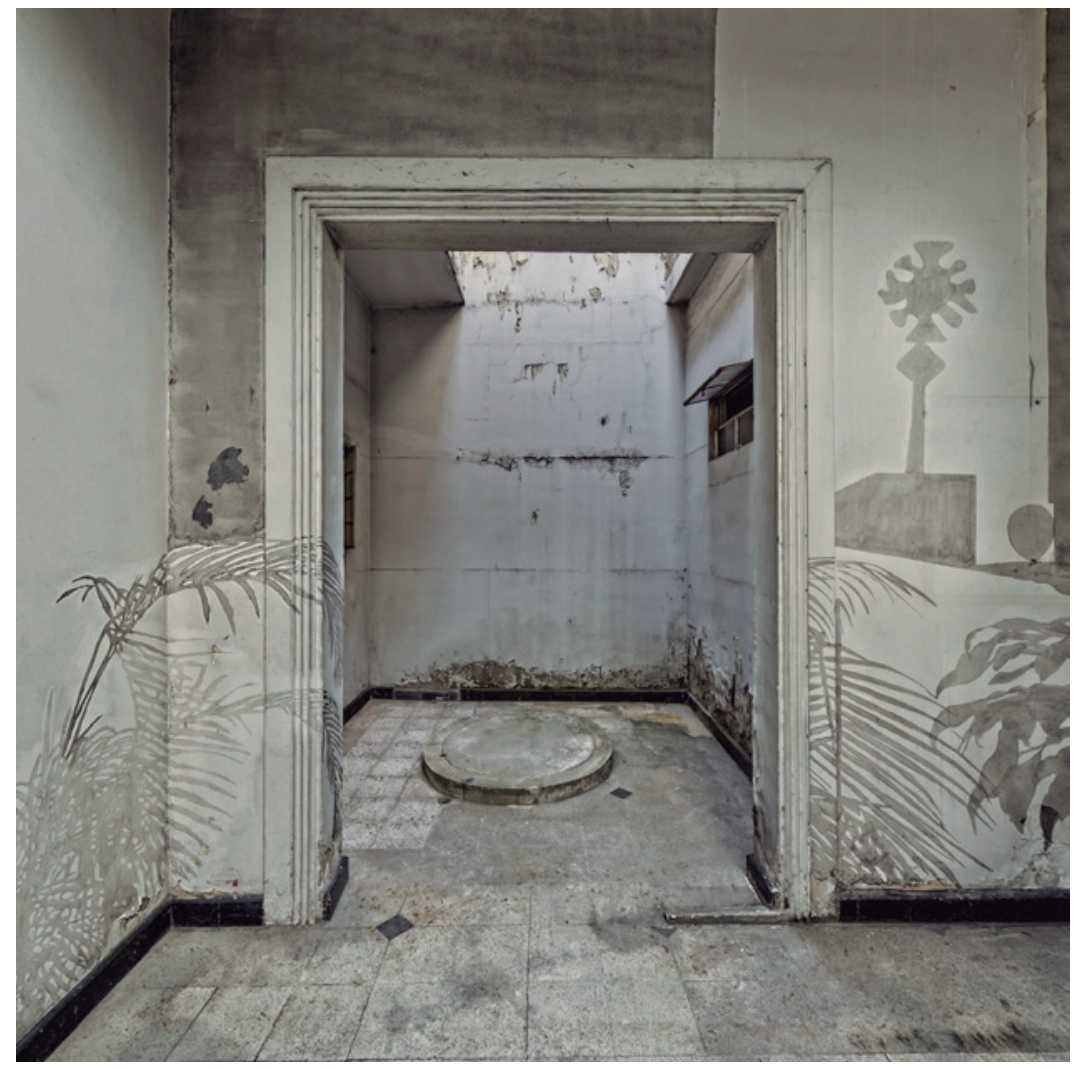

No hay pura luz

ni sombra en los recuerdos:

éstos se hicieron cárdena ceniza

o pavimento sucio

de calle atravesada por los pies de las gentes

que sin cesar salía y entraba en el mercado

Pablo Neruda (1904 - 1973) 